\title{
Comparison of Reconstruction Algorithms for Decreasing the Exposure Dose During Digital Tomosynthesis for Arthroplasty: a Phantom Study
}

\author{
Tsutomu Gomi $^{1}$ • Rina Sakai $^{1}$ - Masami Goto ${ }^{1} \cdot$ Yusuke Watanabe $^{1} \cdot$ Tohoru Takeda $^{1}$. \\ Tokuo Umeda ${ }^{1}$
}

Published online: 4 March 2016

(C) Society for Imaging Informatics in Medicine 2016

\begin{abstract}
To explore the possibility of decreasing the radiation dose during digital tomosynthesis (DT) for arthroplasty, we compared the image qualities of several reconstruction algorithms, such as filtered back projection (FBP) and two iterative reconstruction (IR), methods maximum likelihood expectation maximization (MLEM) and the simultaneous iterative reconstruction technique (SIRT) under different radiation doses. The three algorithms were implemented using a DT system and experimentally evaluated by contrast-to-noise ratio (CNR), artifact spread function (ASF), and power spectrum measurements on a prosthesis phantom. The CNR and ASF data were statistically analyzed by a one-way analysis of variance. The effectiveness of each technique for enhancing the visibility of the prosthesis phantom was quantified by the CNR (reference dose vs. $20 \%$ reduced dose in FBP, $P=0.62$; reference vs. $37 \%$ reduced dose in FBP, $P=0.16$; reference vs. $55 \%$ reduced dose in FBP, $P<0.05$; reference vs. $20 \%$ reduced dose in IR, $P=0.92$; reference vs. $37 \%$ reduced dose in IR, $P=0.40$; reference vs. $55 \%$ reduced dose in IR, $P<0.05$ ) and ASF (reference dose vs. $20 \%$ reduced dose in FBP, $P=0.25$; reference vs. 37 and $55 \%$ reduced dose in FBP, $P<0.05$; reference vs. $20 \%$ reduced dose in IR, $P=0.16$; reference vs. 37 and $55 \%$ reduced dose in IR, $P<0.05$ ). The power spectra under the reference and reduced doses are equivalent. In this phantom study, the radiation dose of the reference dose could be decreased by $20 \%$ with FBP and IR for consideration of common factors.
\end{abstract}

Tsutomu Gomi

gomi@kitasato-u.ac.jp

1 School of Allied Health Sciences, Kitasato University, Kitasato 1-15-1 Minami-ku, Sagamihara, Kanagawa 252-0373, Japan
Keywords Tomosynthesis $\cdot$ Arthroplasty $\cdot$ Radiation dose

\section{Introduction}

Digital tomosynthesis (DT) combines the benefits of digital imaging [1-17] with the tomographic benefits of computed tomography. The DT approach can be easily implemented in conjunction with radiography at reduced radiation doses and cost. DT can provide three-dimensional (3D) structural information.

Artifacts deteriorate the image quality by reducing the contrast and obscuring details, thereby hindering the detection of structures of interest and possibly leading to misdiagnosis. In the presence of metallic joint prosthesis or osteosynthetic materials, the metal implant and the interfaces between the implant, the dose, and the surrounding tissue should be evaluated. Hematoma or inflammation in the adjacent soft tissue must also be ruled out. However, artifacts greatly complicate the evaluation of these features, frequently rendering the images uninterpretable by conventional image reconstruction, even when hard convolution kernels (Ramp or Shepp-Logan (SL) filter kernel) are used such as filtered back projection (FBP) [14]. In DT, artifacts occur as very low signals along the sweep direction around the edges of highly attenuating materials such as metal prosthesis or osteosynthetic materials. These artifacts are predominantly caused by the reconstruction of very low-level signals in the shadow of the highly attenuating object. The limited sweep angle also contributes but to a much lesser degree. Some improvement techniques are suggested to overcome these FBP problems $[8,16]$.

Besides FBP, the iterative reconstruction (IR) method has been explored in DT for arthroplasty [18]. IR was found to effectively decrease quantum noise and radiation exposure and may improve the image quality over the conventional 
FBP technique. However, this previous report was limited to comparisons of FBP and algebraic IR and the simultaneous iterative reconstruction technique (SIRT) [19] and the evaluations of a simple prosthesis and contrast-detail phantom under constant radiation dose. Therefore, it is necessary to evaluate the optimal radiation dose and image quality in prosthesis imaging of each reconstitution methods.

Here, we explore the following three reconstruction techniques: conventional FBP, statistical IR - maximum likelihood expectation maximization (MLEM) [20], and an algebraic reconstruction technique (i.e., SIRT). We evaluate and compare the characteristics of the reconstructed images and the possible reduction in the radiation dose, applying the FBP, SIRT, and MLEM algorithms to prosthesis phantoms. The algorithms were implemented using a DT system and experimentally evaluated against measurements on a prosthesis phantom.

\section{Materials and Methods}

\section{Phantom Specifications}

To evaluate the image quality (implant and artificial bone introduced the artifacts and contrast, respectively), we immersed a prosthesis phantom containing an implant in the center of a polymethyl methacrylate case filled with water (case dimensions $\varphi 200 \times 300 \mathrm{~mm}$; Fig. 1). In phantom experiments, water is often used as the substitute for soft tissues. The area of the phantom that was filled with water was considered to be the composition which simulated soft tissue. The phantom was an artificial bone (orthopaedic humerus models (model normal anatomy, canal diameter $9 \mathrm{~mm}$, overall length 300 mm), Pacific Research Laboratories, Inc, WA, USA). The implant was a TRIGEN Humeral Nails Proximal Straight (model 38153000, diameter $8 \mathrm{~mm}$, overall length $160 \mathrm{~mm}$ ), from Smith \& Nephew Orthopaedics KK Inc, Tokyo, Japan. In the prosthesis phantom, we assumed an internal fracture fixation (intramedullary fracture fixation) simulating a humeral proximal fracture. The prosthetic phantoms were designed to evaluate the reconstruction quality of in-focus plane and out-of-plane images.

\section{DT System}

The DT system (SonialVision Safire II; Shimadzu Co., Kyoto, Japan) consisted of an X-ray tube with a 0.6-mm focal spot and $362.88 \times 362.88-\mathrm{mm}$ digital flat-panel detector composed of amorphous selenium. Each detector element was sized as $150 \times 150 \mu \mathrm{m}^{2}$. Tomography was performed linearly with a total acquisition time of $6.4 \mathrm{~s}$ (reference radiation dose $80 \mathrm{kVp}, 250 \mathrm{~mA}, 16 \mathrm{~ms} / \mathrm{view}$; effective dose, in accordance with the International Commission on Radiological Protection
(ICRP) $0.54 \mathrm{mSv}$ (ICRP 103); $20 \%$ reduced radiation dose 80 $\mathrm{kVp}, 250 \mathrm{~mA}, 14 \mathrm{~ms} / \mathrm{view}, 0.42 \mathrm{mSv} ; 37 \%$ reduced radiation dose $80 \mathrm{kVp}, 250 \mathrm{~mA}, 10 \mathrm{~ms} / \mathrm{view}, 0.33 \mathrm{mSv} ; 55 \%$ reduced radiation dose $80 \mathrm{kVp}, 250 \mathrm{~mA}, 7 \mathrm{~ms} /$ view, $0.24 \mathrm{mSv})$. The acquisition angle was $40^{\circ}$. The effective dose was calculated by Monte Carlo-based software (PCXMC version 2.0; Radiation and Nuclear Safety Authority, Helsinki, Finland) [21]. The reference radiation dose was the dose generally used in clinical practice (the clinical task was to assess the prosthesis). To sample the projection images during a single tomographic pass (74 projections) and reconstruct tomograms of the desired height, we used a $(1024 \times 1024)$ matrix with 12 bits per image. The reconstructed images $(0.279 \mathrm{~mm} /$ pixel $)$ were obtained at $1-\mathrm{mm}$ reconstruction intervals using an antiscatter grid (focused type, grid ratio 12:1). The source to isocenter and isocenter to detector distances were 980 and $1100 \mathrm{~mm}$, respectively (the latter is equivalent to filtration through 3.0-mm aluminum).

\section{The Reconstruction Algorithm}

The impulse shape of the reconstructed image was restored by two-dimensional (2D) image filtering, which multiplies the Fourier transform by a Ramp or SL filter kernel. The FBP algorithm generally yields precise $3 \mathrm{D}$ reconstruction images [14]. In this study, the FBP images were reconstructed by a conventional Ramp or an SL filter kernel.

Unlike the one-step operation of back projection and FBP algorithms, IR algorithms perform a recursive reconstruction [22]. IR iteratively updates the unknown linear attenuation coefficients by minimizing the error between the measured and calculated projection data.

The original method in this family of algebraic reconstruction techniques (ARTs) [19] has been already characterized. ART rapidly converges because it updates the linear attenuation coefficients from a single projection value at each time point. However, if the inverse problem is severely ill-posed, as occurs in a limited angle reconstruction, the least squares solution can contain considerable noise. Several improved variations of ART have been proposed. For instance, ART can be modified for compatibility with other methods such as SIRT (which performs adding and multiplying operations, we chose adding type in this study) depending on the volume of projection data and method used to update the current estimation. MLEM methods consisting of two steps per iteration (i.e., a forward step for modeling the DT acquisition process and a backward step for updating the reconstructed object) have also been proposed. MLEM and SIRT (adding type) are applied iteratively, such that the reconstructed volume projections (computed from an image formation model) resemble the experimental projections. In the present study, we maximize the contrast and minimize the artifacts by ten iterations of MLEM and SIRT (adding type). The FBP, MLEM, and SIRT (adding 

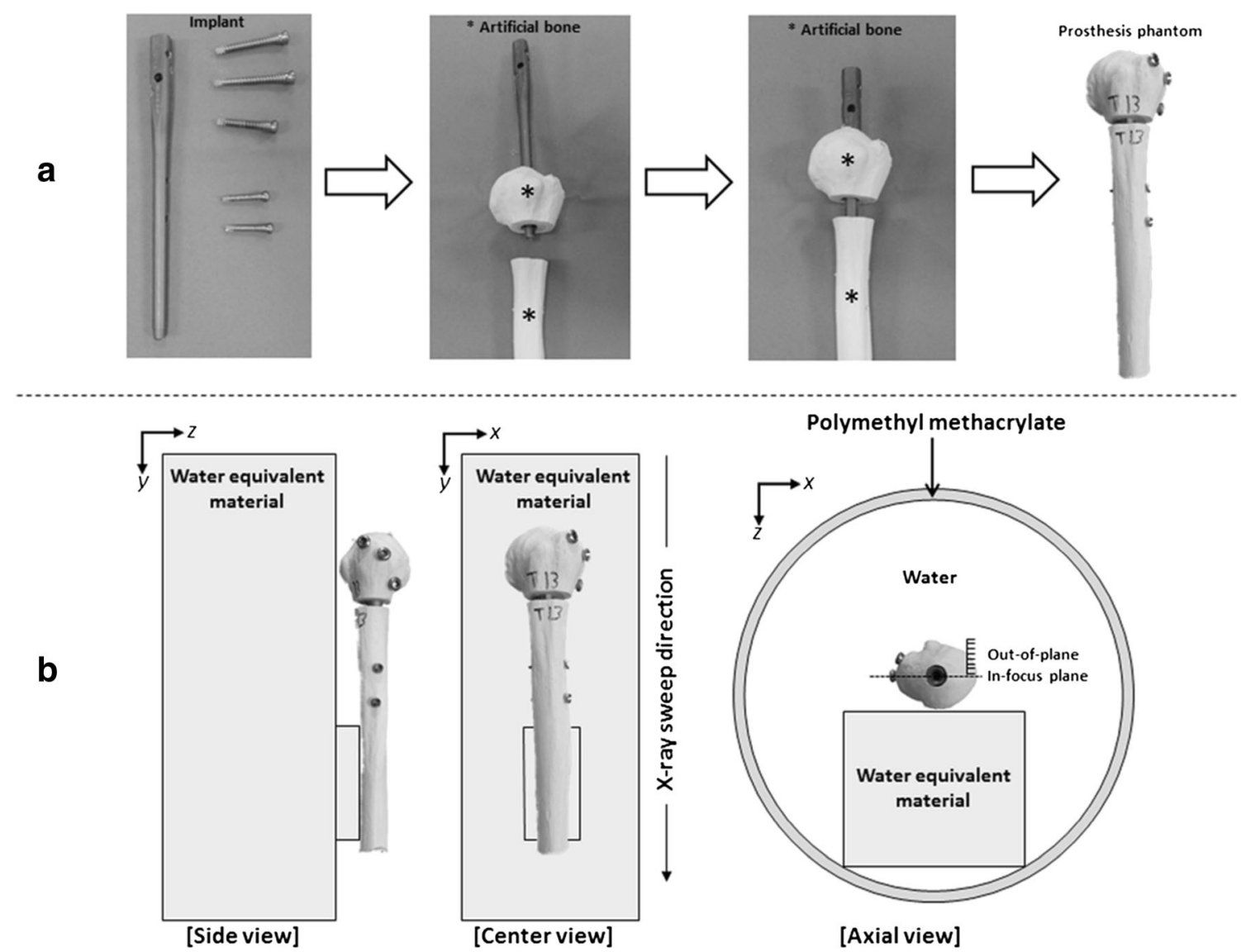

Fig. 1 a Photograph of the prosthesis phantom used in this study. b Geometric distribution of the prosthesis phantom. The phantom is placed in the center of the cylindrical water tank

type) image reconstruction calculations were implemented in MATLAB (Mathworks, Natick, MA, USA) [23]. The data for the reconstruction were real projection data acquired by a DT system.

\section{Evaluation}

To evaluate the effects of image quality (i.e., contrast and artifacts) on the reconstructed image features in the in-focus plane, we calculated the intensity profile and measured its contrast-to-noise ratio (CNR). To evaluate the effects of artifacts on the reconstructed image features in the adjacent outof-plane area, we calculated an artifact spread function (ASF). For DT acquisition, the phantom was arranged parallel to the detector plane.

We compared the intensity profiles of different reconstruction methods and radiation doses in the in-focus plane. In these comparisons, we evaluated the regions of the stem containing artifacts after processing by each technique. The intensity profiles were set to 132 pixels.

To quantitatively evaluate the reconstructed image quality (contrast), we calculated the image contrast derived from the CNR of the selected two features (area of femoral head and diaphysis) and then determined the low-contrast detectability
$(40 \times 20$-pixel region) in the in-focus plane for the detailed detection of bone changes. The CNR is defined as follows:

$\mathrm{CNR}=\frac{N_{1}-N_{0}}{\sigma_{0}}$

where $N_{1}$ and $N_{0}$ are the mean pixel values of the object (artificial bone) and background, respectively, and $\sigma_{0}$ is the standard deviation of the background pixel values. The parameter $\sigma_{0}$ includes not only the photon statistics and electronic noise in the results but also the structural noise that can obscure the object.

$\mathrm{Wu}$ et al. proposed an ASF metric for quantifying artifacts observed in planes outside the focus image plane [20]. These artifacts, which resemble real features, are generated from real features located within the focus image plane. The ASF measurement reflects the ability of DT to differentiate features that are superimposed along the tomographic slice direction. The ASF of artifacts exhibited in the image plane is given by

$\mathrm{ASF}=\frac{N_{\text {artifact }}(z)-N_{\mathrm{BG}}(z)}{N_{\text {artifact }}\left(z_{0}\right)-N_{\mathrm{BG}}\left(z_{0}\right)}$

where $z_{0}$ and $z$ are the locations of the real features in the infocus and out-of-plane images, respectively. $N_{\text {artifact }}\left(z_{0}\right)$ and 
$N_{\mathrm{BG}}\left(z_{0}\right)$ are the regions of interest (ROIs) for average pixel intensities of the features and background, respectively, in the in-focus-plane image. $N_{\text {artifact }}(z)$ and $N_{\mathrm{BG}}(z)$ are the corresponding ROIs in the out-of-plane image. The ROI size in the evaluation of all features was $4 \times 4$ pixels.

The effects of contrast and artifact were assessed by a oneway analysis of variance and multiple comparison (i.e., the Tukey-Kramer test). In these tests, we compared the CNR values of the reconstruction algorithms at different radiation doses. The sample numbers were 16 (for CNR) and 152 (for ASF). The statistical analysis was performed in SPSS for Windows, version 21.0 (SPSS Inc., Chicago, IL, USA). Probability $(P)$ values $<0.05$ were assumed as statistically significant.

Frequency analysis measures the spatial frequency components in DT images. For a frequency analysis of the DT images acquired under the reference and reduced radiation doses, we computed the normalized power spectrum [24], which shows the contribution of each component in the image construction. Compared with the spectrum of an imperfect image, it also reveals image deterioration. The power spectral analysis was performed over the whole image.

\section{Results}

The reconstructed images of the prosthetic phantom obtained by conventional FBP (with Ramp and SL kernels), SIRT (addition type), and MLEM are presented in Fig. 2. Figure 3 shows the intensity profiles of the stem generated by the conventional FBP (with Ramp and SL kernels), SIRT (addition type), and MLEM. The features in DT images generated by the SIRT and
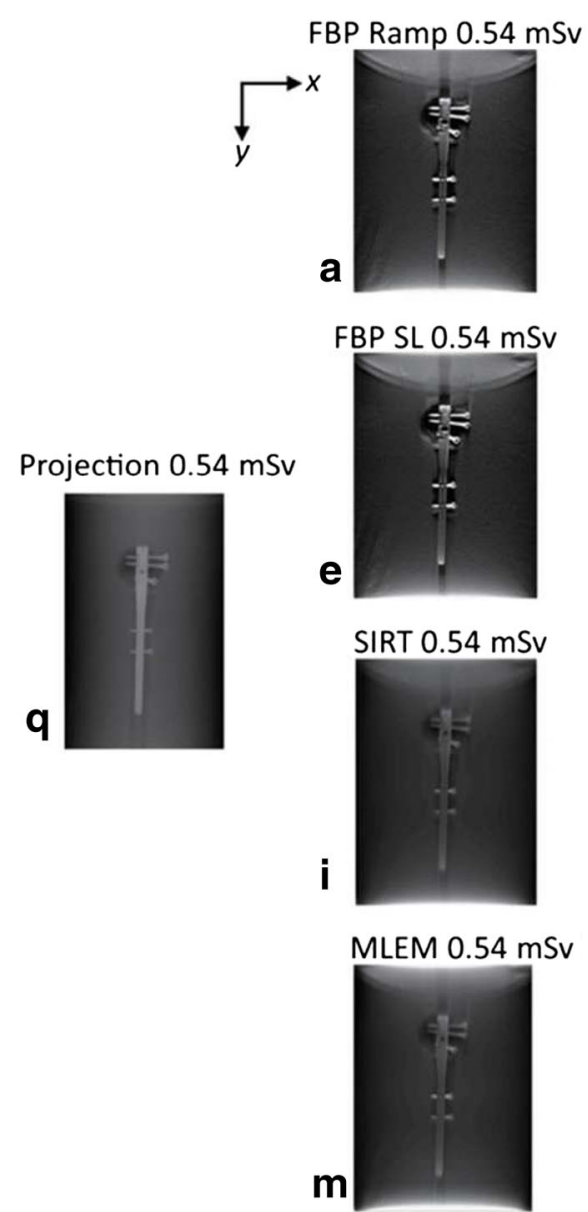

Fig. 2 Comparisons between the images obtained from tomosynthesis and the imaging algorithms of conventional filtered back projection (FBP), simultaneous iterative reconstruction technique (SIRT), and maximum likelihood expectation maximization (MLEM) in the in-focus plane. The window width and level of the prosthesis phantom display are varied to visually compare the contrast and background gray level. The $\mathrm{X}$-ray source is moved vertically along the image. The signal intensity profiles were measured in the displayed areas. FBP (Ramp) a reference radiation dose, b $20 \%$ reduced dose, c $37 \%$ reduced dose, and d $55 \%$
FBP Ramp $0.42 \mathrm{mSv}$ b

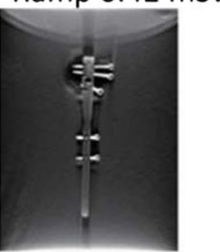

FBP SL $0.42 \mathrm{mSV}$

$\mathbf{f}$
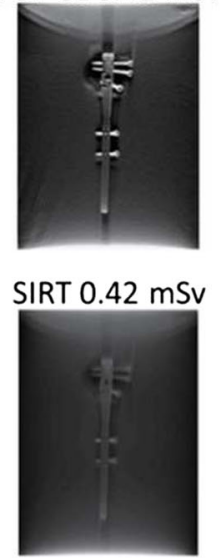

MLEM $0.42 \mathrm{mSV}$

n

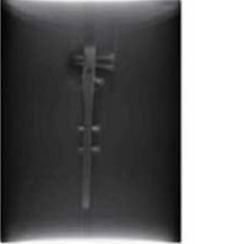

FBP Ramp $0.33 \mathrm{mSv}$

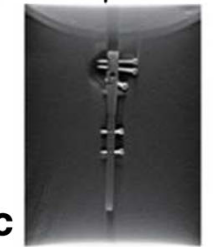

FBP SL $0.33 \mathrm{mSV}$
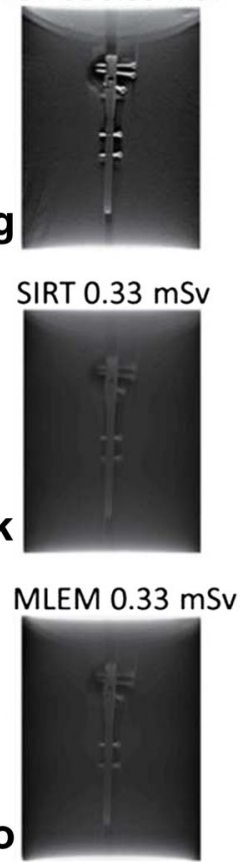

FBP Ramp $0.24 \mathrm{mSv}$

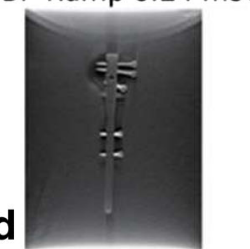

FBP SL $0.24 \mathrm{mSV}$
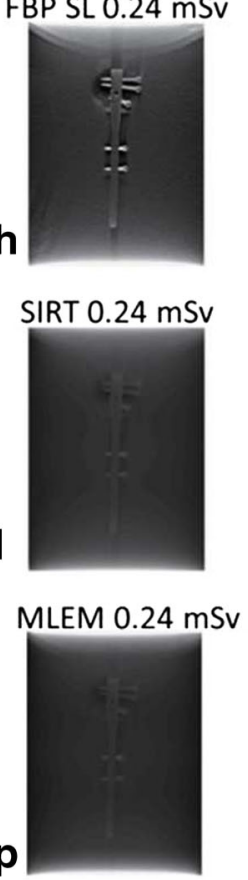

reduced dose; FBP (SL) e reference radiation dose, $\mathbf{f} 20 \%$ reduced dose, $\mathbf{g}$ $37 \%$ reduced dose, and $\mathbf{h} 55 \%$ reduced dose; SIRT (adding type) i reference radiation dose, j $20 \%$ reduced dose, k $37 \%$ reduced dose, and $\mathbf{l} 55 \%$ reduced radiation dose; and MLEM $\mathbf{m}$ reference dose, $\mathbf{n}$ $20 \%$ reduced dose, $\mathbf{0} 37 \%$ reduced dose, p $55 \%$ reduced radiation dose, and $\mathbf{q}$ reference radiation dose of projection images $(0.54 \mathrm{mSv})$. The reference dose $(0.54 \mathrm{mSv})$ and its reductions by 20,37 , and $55 \%(0.42$, 0.33 , and $0.24 \mathrm{mSv}$, respectively) are the same in all methods 

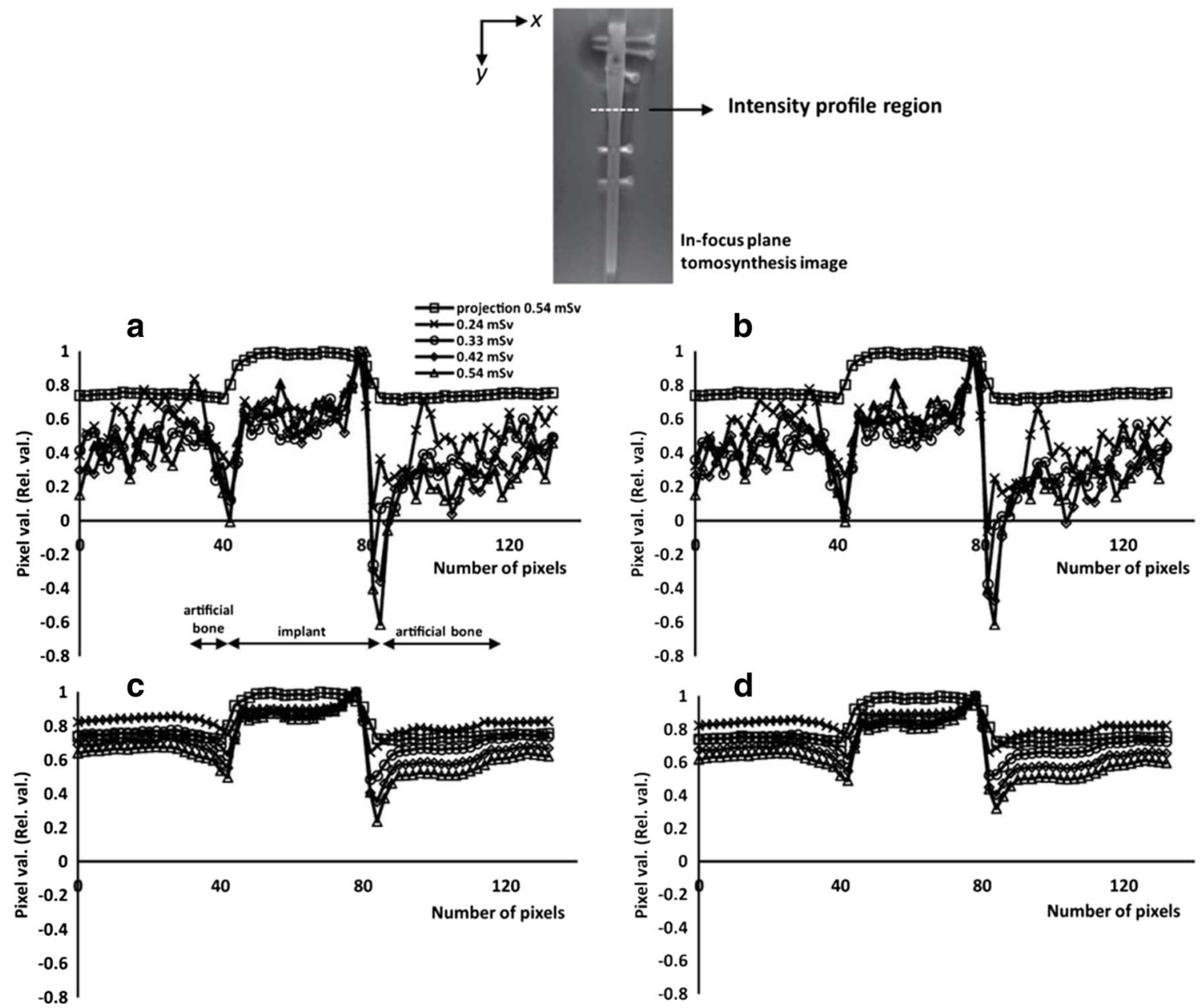

Fig. 3 Comparisons of the intensity profiles obtained by tomosynthesis in the in-focus plane under different radiation doses. The pixel values were normalized by the mean of each intensity profile. a FBP (Ramp), b FBP (SL), c SIRT (adding type), and d MLEM

MLEM methods contained reduced artifacts in the vertical direction (X-ray sweep direction). In particular, artifacts were reduced in the peripheral regions of the prosthetic phantom.

Figure 4 presents the ROIs in the prosthetic phantom and plots the CNR results. The contrasts (ROI 1 femoral diaphysis) in FBP and IR yielded approximately equivalent CNRs for the reference radiation dose $(0.54 \mathrm{mSv})$ and the $37 \%$ reduced radiation dose $(0.33 \mathrm{mSv})$. For the FBP, a CNR value of the same radiation dose became a similarity with radiation dose reduction. For the IR, the $\mathrm{CNR}$ difference of the same radiation dose was constant. Because of contrasts (ROI 2 femoral head) in FBP and IR, the CNR value in the same radiation dose became a similarity with radiation dose reduction (Fig. 4).

In FBP and IR, the contrast differences between the reference radiation dose (FBP $4.24 \pm 0.81$, IR $8.41 \pm 0.45)$ and the $20 \%$ reduced dose (FBP $3.24 \pm 0.60$, IR $7.54 \pm 0.84$ ) and the reference dose and the $37 \%$ reduced dose (FBP 2.41 \pm 0.48 , IR $6.09 \pm 1.03)$ were also statistically insignificant [FBP $(P=0.62$, difference $0.99,95 \% \mathrm{CI}-1.41$ to 3.40$)$, IR $(P=0.92$, difference $0.87,95 \% \mathrm{CI}-3.35$ to 5.10$)$ in the former case; FBP $(P=0.16$, difference $1.83,95 \%$
CI -1.57 to 4.24$)$, IR $(P=0.40$, difference $2.31,95 \%$ CI -1.91 to 6.54$)$ in the latter]. However, the contrast difference between the reference radiation dose and the $55 \%$ reduced dose (FBP $1.34 \pm 0.21$, IR $3.60 \pm 1.43$ ) was statistically significant in the IR [FBP $(P<0.05$, difference $2.90,95 \%$ CI 0.49 to 5.31$)$, IR $(P<0.05$, difference $4.81,95 \%$ CI 0.58 to 9.04$)$ ].

In FBP and IR, the artifact differences between the reference radiation dose $(\mathrm{FBP} 0.89 \pm 0.03$, IR $0.77 \pm 0.03)$ and the $37 \%$ reduced dose (FBP $1.05 \pm 0.02$, IR $0.93 \pm 0.01)$ and the reference dose and the $55 \%$ reduced dose (FBP $1.20 \pm 0.03$, IR $0.91 \pm 0.03)$ were also statistically significant [FBP $(P<0.05$, difference $0.16,95 \% \mathrm{CI}-0.25$ to -0.06$)$, IR $(P<0.05$, difference $0.15,95 \% \mathrm{CI}-0.25$ to -0.05$)$ in the former case; FBP $(P<0.05$, difference $0.31,95 \% \mathrm{CI}-0.40$ to -0.21$)$, IR $(P<0.05$, difference $0.13,95 \% \mathrm{CI}-0.24$ to $-0.03)$ in the latter]. However, the artifact difference between the reference radiation dose and the $20 \%$ reduced dose (FBP $0.96 \pm 0.01$, IR $0.86 \pm 0.02)$ was statistically insignificant in the IR [FBP $(P=0.25$, difference $0.06,95 \% \mathrm{CI}-0.16$ to 0.02), IR $(P=0.16$, difference $0.08,95 \% \mathrm{CI}-0.18$ to 0.02$)]$. 


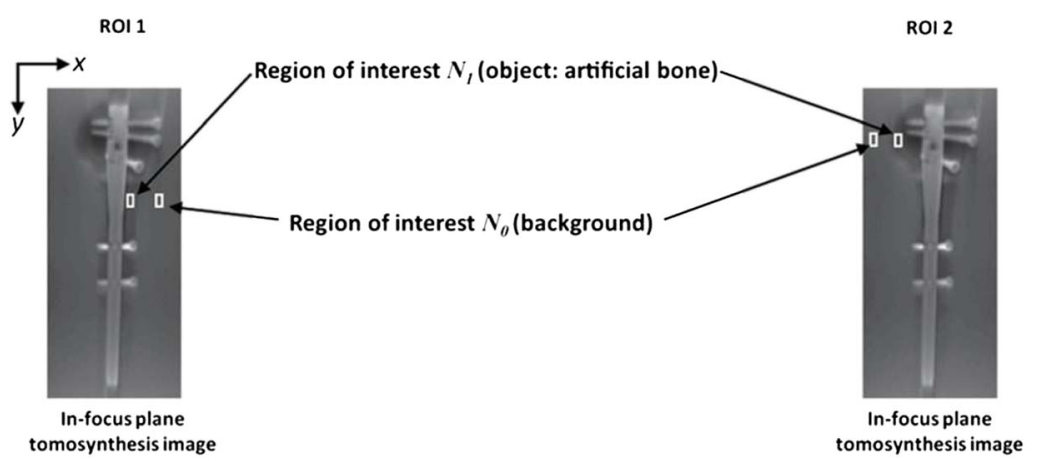

CNR ROI 1

CNR ROI 2
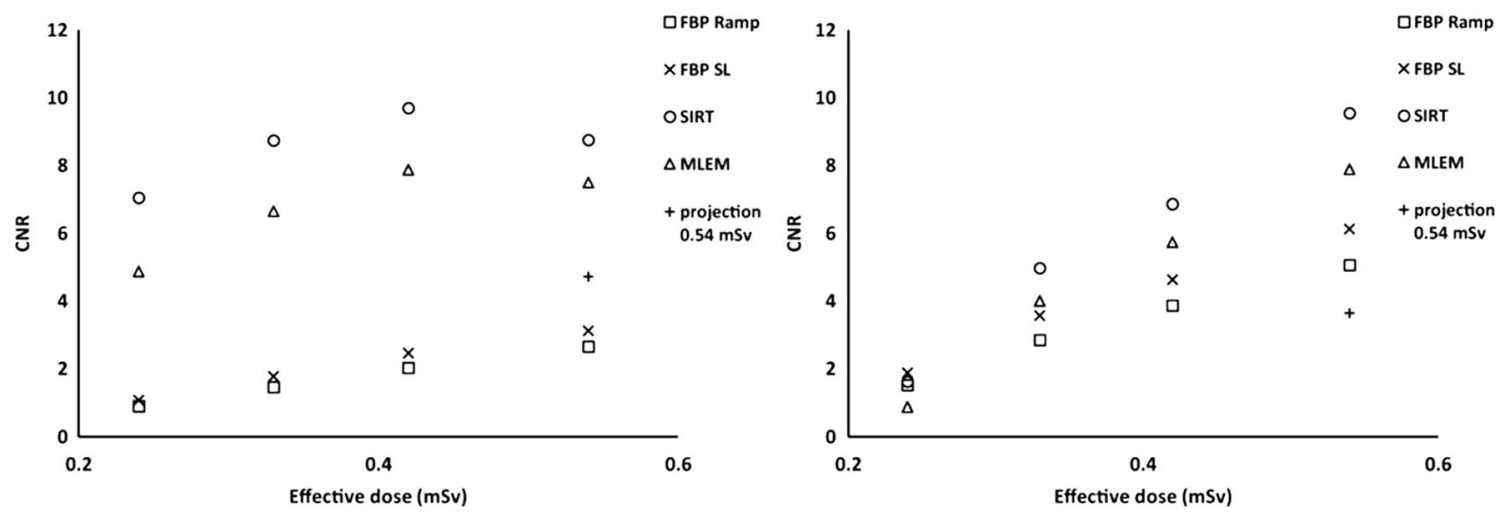

Fig. 4 Comparisons of the contrast-to-noise ratios (CNRs) in the in-focus plane images obtained by tomosynthesis under different radiation doses. Image contrast derived from the CNR of the selected two features. Top image indicates the object and background areas of the CNR measurements

Figure 5 presents the ROIs in the prosthetic phantom and plots the ASF results. The SIRT (adding type) and MLEM methods removed the highest number of artifacts. The artificial image tended to need enlargement at low radiation doses. The image quality was always higher under the reference radiation dose than under the reduced radiation doses, regardless of method.

Comparing the intensity profiles, CNRs, and ASFs under different radiation doses, we find that in FBP and IR, to preserve the high contrast and low artifacts (consider the common factor of CNR and ASF from statistical tests) of the reference dose image, the radiation exposure must be under $20 \%$ reduced dose.

Figure 6 plots the power spectra under the reference radiation dose and $20 \%$ reduced radiation doses. The power values of the spatial frequency components are comparable among the three doses, implying that lower radiation doses largely preserve the image integrity.

\section{Discussion}

Our empirical results, obtained by various methods, clearly demonstrate the feasibility of reducing the reference radiation dose by $20 \%$ in IR and FBP methods.
From the intensity profiles, CNRs, and ASFs presented in Figs. 3, 4, and 5, we can quantitatively evaluate the efficiency of the DT technique. In the CNR experiment of the FBP- and IR-obtained images, the detectable contrast was very similar under the reference radiation dose and the $37 \%$ reduced dose (however, under dose reductions of 37 and $55 \%$, the artifacts increased). We think that to consider the common factor of each dose image, the radiation exposure must be under $20 \%$ reduced dose. These results suggest that we can reduce the DT radiation dose administered to patients.

Rather than assuming even noise distribution across the entire image, SIRT selectively identifies and then subtracts noise from the image using a mathematical model based on an algebraic matrix. MLEM identifies the reconstructed image that maximizes the likelihood of observing the particular projection measurements [20]. Because each iteration of the MLEM algorithm amplifies the high-frequency noise in the data, a reduction in the number of iterations may optimize the detection of low-contrast objects in this method.

In general, image artifacts are caused by a loss of the largest normal contributions from artifact-free voxels. Consequently, the contributions of these voxels are reduced from their original values. Conversely, a voxel receiving a single abnormal contribution while retaining all other contributions (including 


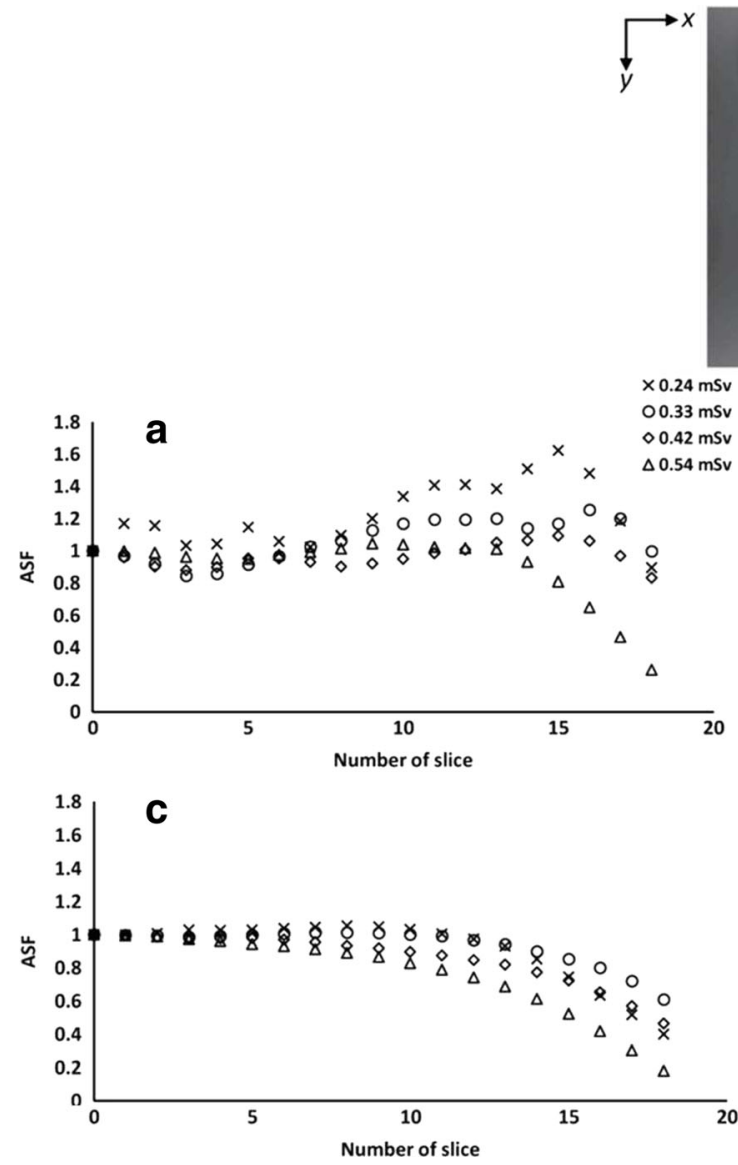

Fig. 5 Plots of the artifact spread function (ASF) versus the slice number from the in-focus plane for tomosynthesis images acquired under different radiation doses. Bottom right image indicates the artifact and

its largest normal contribution) tends to exhibit higher values than its neighboring artifact-free voxels. In this case, the
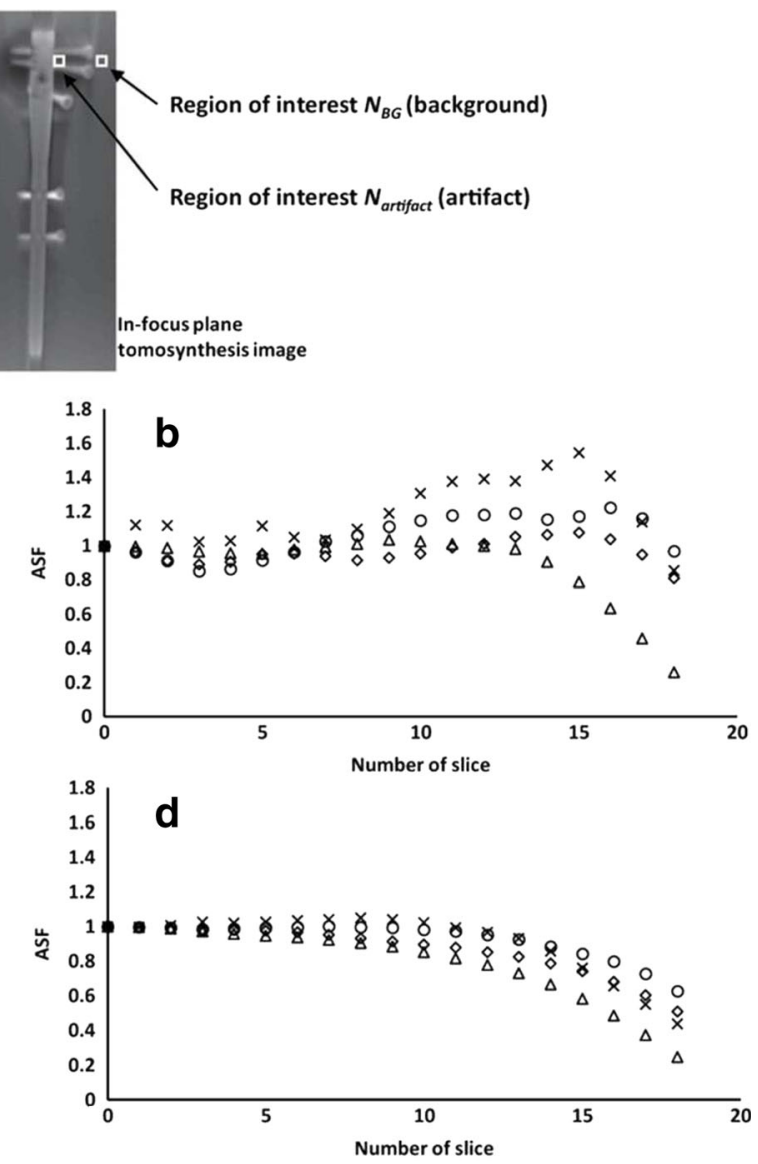

background areas of the ASF measurements. a FBP (Ramp), b FBP (SL), c SIRT (adding type), and d MLEM

artifact-free voxels are more noticeable against the background than the natural voxels. Although this phenomenon

Fig. 6 Power spectra of tomosynthesis images acquired in the in-focus plane under reference and reduced radiation doses. Reference radiation dose versus a $20 \%$ reduced radiation dose in FBP (Ramp), b $20 \%$ reduced dose in FBP (SL), c $20 \%$ reduced dose in SIRT (adding type), and $\mathbf{d}$ $20 \%$ reduced dose in MLEM. Reference and $20 \%$ reduced doses are 0.54 and $0.42 \mathrm{mSv}$, respectively
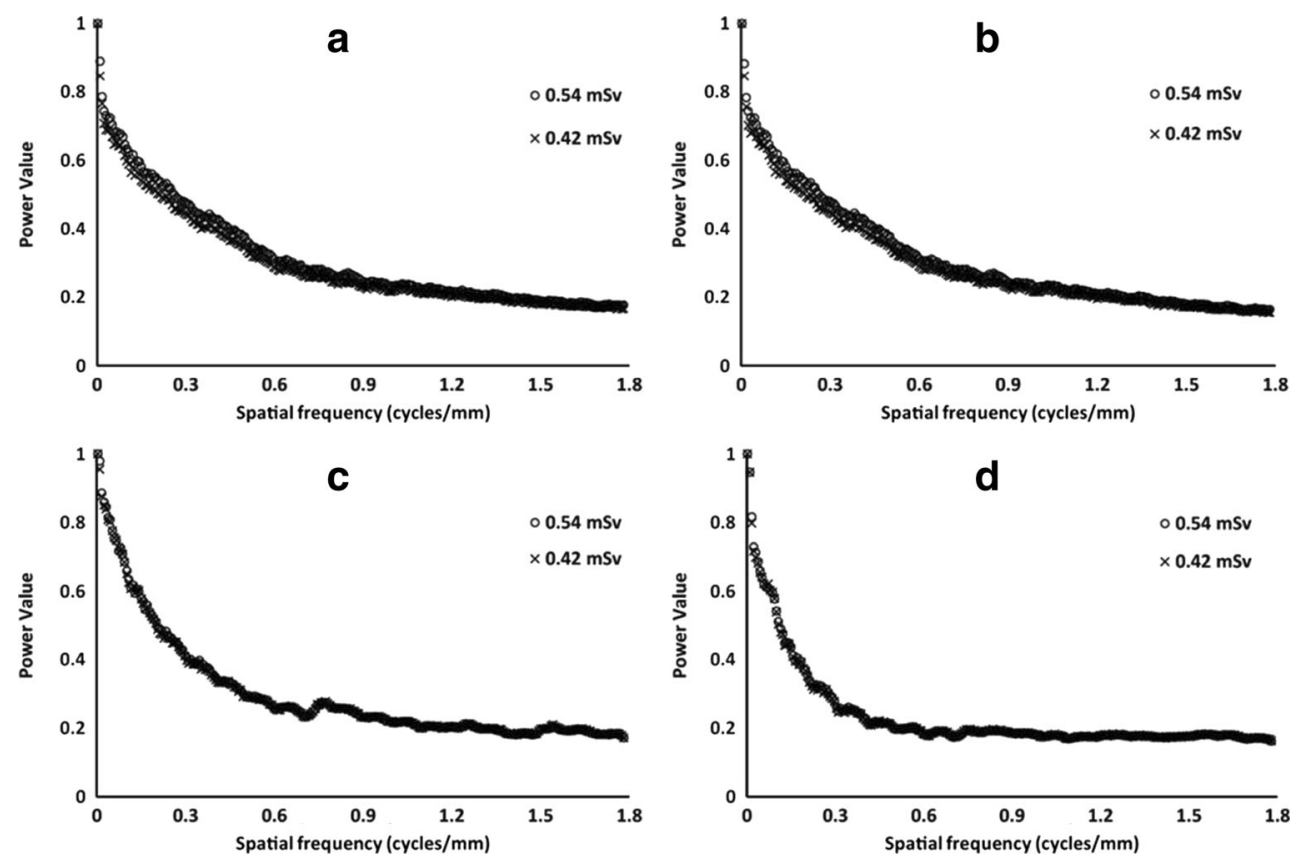
compromises the FBP technique, such artifacts are very conspicuous in comparisons with artifact-free images.

Quantum noise seriously degrades the resolution of radiographic contrasts. This noise increases as the inverse square root of the X-ray exposure dominates the noise at low radiation levels. Quantum noise limits the technical practicality of reducing the radiation doses in DT systems below those of conventional tomography. However, if quantum noise can be ignored, tomograms can be obtained under the technical specifications of radiography. Moreover, by reconstructing numerous image slices from a single low-dose image data acquisition, DT overcomes the difficulties of geometric tomography.

Most studies evaluate a technical report about prosthesis imaging under constant radiation dose [8, 15, 16, 18]. We think that it is useful that we investigate radiation dose and its relation with image quality and we were able to suggest optimal radiation dose. We believe that the results of this paper will become a guideline for imaging acquisition condition determination when considering of image quality.

Our phantom study has some limitations. The materials constituting the prosthesis phantom only simulate the arthroplasty; i.e., we tested artificial bone and implant. Our results should provide useful reference data for physicians desiring to reduce radiation exposure to their patients.

\section{Conclusion}

We compared various DT reconstruction methods in a prosthesis phantom study. We found that in FBP and IR, the radiation levels can be decreased by $20 \%$ (relative to the reference dose) without largely compromising the image quality.

Acknowledgments We wish to thank Mr. Kazuaki Suwa at the Department of Radiology, Dokkyo Medical University Koshigaya Hospital, for the support on the experiment.

\section{References}

1. Ziedses des Plante BG: Eine neue methode zur differenzierung in der roentgenographie (planigraphie). Acta Radiol 13:182-192, 1932

2. Miller ER, McCurry EM, Hruska B: An infinite number of laminagrams from a finite number of radiographs. Radiology 98: 249-255, 1971

3. Grant DG: Tomosynthesis. A three-dimensional radiographic imaging technique. IEEE Trans Biomed Eng 19:20-28, 1972

4. Baily NA, Lasser EC, Crepeau RL: Electrofluoro-plangigraphy. Radiology 107:669-671, 1973

5. Kruger RA, Nelson JA, Ghosh-Roy D, Miller FJ, Anderson RE, Liu PY: Dynamic tomographic digital subtraction angiography using temporal filteration. Radiology 147:863-867, 1983
6. Sone S, Kasuga T, Sakai S, Aoki J, Izuno I, Tanizaki Y, Shigeta H, Shibata K: Development of a high-resolution digital tomosynthesis system and its clinical application. Radiographics 11:807-822, 1991

7. Sone S, Kasuga T, Sakai F, Kawai T, Oguchi K, Hirano H, Li F, Kubo K, Honda T, Hniuda M, Takemura K, Hosoba M: Image processing in the digital tomosynthesis for pulmonary imaging. Eur Radiol 5:96-101, 1995

8. Machida H, Yuhara T, Mori T, Ueno E, Moribe Y, Sabol JM: Optimizing parameters for flat-panel detector digital tomosynthesis. Radiographics 30:549-562, 2010

9. Vikgren J, Zachrisson S, Svalkvist A, Johnsson AA, Boijsen M, Flinck A, Kheddache S, Båth M: Comparison of chest tomosynthesis and chest radiography for detection of pulmonary nodules: human observer study of clinical cases. Radiology 249 : 1034-1041, 2008

10. Skaane P, Bandos AI, Gullien R, Eben EB, Ekseth U, Haakenaasen U, Izadi M, Jebsen IN, Jahr G, Krager M, Niklason LT, Hofvind S, Gur D: Comparison of digital mammography alone and digital mammography plus tomosynthesis in a population-based screening program. Radiology 267:47-56, 2013

11. Stiel G, Stiel LG, Klotz E, Nienaber CA: Digital flashing tomosynthesis: a promising technique for angiographic screening. IEEE Trans Med Imaging 12:314-321, 1993

12. Duryea J, Dobbins JT, Lynch JA: Digital tomosynthesis of hand joints for arthritis assessment. Med Phys 30:325-33, 2003

13. Niklason LT, Christian BT, Niklason LE, Kopans DB, Castleberry DE, Opsahl-Ong BH, Landberg CE, Slanetz PJ, Giardino AA, Moore R, Albagli D, DeJule MC, Fitzgerald PF, Fobare DF, Giambattista BW, Kwasnick RF, Liu J, Lubowski SJ, Possin GE, Richotte JF, Wei CY, Wirth RF: Digital tomosynthesis in breast imaging. Radiology 205:399-406, 1994

14. Dobbins III, JT, Godfrey DJ: Digital X-ray tomosynthesis: current state of the art and clinical potential. Phys Med Biol 48:R65-106, 2003

15. Gomi T, Hirano H: Clinical potential of digital linear tomosynthesis imaging of total joint arthroplasty. J Digit Imaging 21:312-322, 2008

16. Gomi T, Hirano H, Umeda T: Evaluation of the X-ray digital linear tomosynthesis reconstruction processing method for metal artifact reduction. Comput Med Imaging Graph 33:257-274, 2009

17. White LM, Buckwalder KA: Technical considerations: CT and MR imaging in the postoperative orthopaedic patient. Semin Musculoskelet Radiol 6:5-17, 2002

18. Gomi T: Comparison of metal artifact in digital tomosynthesis and computed tomography for evaluation of phantoms. J Biomed Sci Eng 6:722-731, 2013

19. Gordon R, Bender R, Hermen GT: Algebraic reconstruction techniques (ART) for three-dimensional electron microscopy and X-ray photography. J Theor Biol 29:471-481, 1970

20. Wu T, Stewart A, Stanton M, McCauley T, Phillips W, Kopans DB, et al: Tomographic mammography using a limited number of lowdose cone-beam projection images. Med Phys 30:365-380, 2003

21. Tapiovaara M, Siiskonen T. A Monte Carlo program for calculating patient doses in medical X-ray examinations, 2nd edition. Report No. STUK-A231 STUK 2008; Helsinki, Finland

22. Bleuet P, Guillemaud R, Magnin I, Desbat L: An adapted fan volume sampling scheme for 3D algebraic reconstruction in linear tomosynthesis. IEEE Trans Nucl Sci 3:1720-1724, 2001

23. Mathworks Inc 2014; http://www.mathworks.com/products/ matlab/

24. Rangayyan RM: Biomedical Image Analysis. CRC Press, Florida, 2000, pp 612-621 